\title{
Facial Asymmetry Analysis Based on 3-D Dynamic Scans
}

\author{
Wei Quan, Bogdan J. Matuszewski, Lik-Kwan Shark \\ ADSIP Research Centre \\ University of Central Lancashire \\ Preston, PR1 2HE, U.K. \\ \{WQuan, BMatuszewski1, LShark\}@uclan.ac.uk
}

\begin{abstract}
Facial asymmetry is an important characteristic used in a number of applications. It plays a vital role in human perception of attractiveness and as such has been used in psychology including research on facial expressions evaluation as well as in plastic surgery and orthodontics. It has been also recognized as a biometric feature used for identification and has important applications in detection and quantification of medical disorders, including neurological conditions, such as stroke or Bell's palsy. The current methods for assessing facial asymmetry mainly rely on manual semi-automatic procedures which have significant limitations as they are subjective in nature. This paper presents a computer-based methodology of automatic facial asymmetry analysis which has been used for detection and quantification of facial dysfunctions. The method is based on dynamic 3-D scans of human faces. Using facial sequences from the Hi4D-ADSIP database, the preliminary evaluation results suggest that the proposed method is able to assist in quantification and diagnosis of facial dysfunctions for neurological patients.
\end{abstract}

Keywords - facial dysfunction measurement and diagnosis, facial asymmetry analysis, 3-D dynamic facial database

\section{INTRODUCTION}

Face is an essential medium which reflects a person's emotional and awareness states, cognitive activity, personality or wellbeing. In the past two decades, the analysis of facial data has become of great interest to computer sciences and machine vision communities, which has been exploited in applications such as human computer interaction [1], augmented reality [2], identity authentication [3], expression recognition [4], and medical diagnosis [5]. Facial dysfunction, which relates to many neurological illnesses, is an important cue often used in medical diagnosis, for grading the severity by the trained practitioners. Such assessment has significant limitations as it is based on a subjective scale and does not provide sufficient specificity and sensitivity, especially for detecting neurological conditions in early stages or identifying their subtle changes. Hence, a computer based approach to achieve automatic facial asymmetry analysis has been the goal to improve the efficiency and accuracy of facial dysfunction identification.

Human faces are not perfectly bilaterally symmetric, but the deviation from perfect bilateral symmetry is expected not to be very significant for healthy subjects. Therefore the asymmetry analysis of a human face is often used for direct detection and assessment of facial dysfunctions which are caused by the relevant illnesses [6]. One of the most important tasks on the facial asymmetry analysis is to identify the symmetry plane of human faces. Once it is determined, the asymmetry level of a face can be directly analysed and assessed along the symmetry plane.

Generally speaking, the methods for extracting the symmetry plane of objects can be classified into two categories in terms of data used, namely, 2-D and 3-D approaches. Marola presents a method for detecting the symmetrical structure of the object in 2-D images [7]. It can only be applied to objects that are almost symmetric and requires the estimation of its centre of mass. Masuda et al. describe another 2-D method to determine the symmetry plane of objects [8]. The method uses the correlation with the rotated-reflected image for extracting the symmetric plane of an object without knowing its centroid position. Kiryati et al. develop an approach for detecting local symmetry in grey level images, which is optimised using a probabilistic genetic algorithm [9].

Regarding the methodologies for 3-D facial shapes, Sun and Sherrah introduced a technique for obtaining the symmetry information of the 3-D model based on the extended Gaussian image (EGI) [10]. It measures the degree of reflectional symmetry across a plane by correlation of the orientation histogram with itself after reflection across the plane. To increase efficiency of the method, it only checks planes near the principal axis of the object, since any plane of symmetry is perpendicular to the principal axis if the object is considered as a perfect symmetrical body. The method is difficult to deal with the facial model containing noise. Pan et al. proposed an extension of Sun and Sherrah's method by introducing two extra steps, normal and offset finding [11]. It is claimed that the method is able to handle models with different facial expressions and noise. Benz et al. describe an approach for determination of the symmetry plane for a 3-D data registration problem [12]. In this method, the position of the symmetry plane is determined by superimposing the original 3-D data onto its mirror data which is obtained by a reflection along an initially arbitrary plane. The symmetry plane is then characterised by matching the original face's spatial arrangement with its mirror data.

Since the human face is a three-dimensional object, the approaches for detecting the symmetry plane of the face in 2-D 
images often suffer from the limitations due to viewpoint and lighting variation. 3-D facial surfaces represent the true structure of faces, which is related to the internal anatomical geometry of faces instead of external appearance [13]. Thus the methods using 3-D facial surfaces have potential for greater accuracy than the methods relying on 2-D images, and advantages of analysing facial geometry irrespective of viewpoint and illumination variability. With the recent development in 3-D imaging systems and ever increasing computing power, 3-D facial asymmetry analysis has started attracting a lot of attention.

In this paper, an automatic approach for measuring asymmetry of 3-D faces is presented, which is invariant to arbitrary poses and variations in facial expressions. The proposed method is able to compute a symmetry plane of human faces in 3-D space by combining the knowledge of principal axes and an iterative point registration scheme. It also enables a number of important features points around eyes, nose and mouth to be extracted. These features could be used for segmenting a face into local regions, such as front head, upper face and lower face. Therefore, specific asymmetric assessment for regions of interest instead of the entire facial area is possible. The information of facial articulation (movement) recorded in 3-D dynamic facial sequences is also included in the proposed method to improve the accuracy of the facial asymmetry analysis, whereby the level of facial asymmetry is treated as a function of time in the face movement.

The remainder of this paper is organized as follows. Section 2 briefly introduces the 3-D dynamic facial database used for the evaluation in this work. Section 3 describes the face segmentation prior to the facial asymmetry analysis. Section 4 provides details of extracting the symmetry plane and computing the facial asymmetry. Discussion of the method evaluation is presented in Section 5. Finally, concluding remarks and future work are discussed in Section 6.

\section{3-D DYNAMIC FACIAL DATABASE}

To investigate the feasibility and performance of the proposed method for facial asymmetry analysis, the 3-D facial data need to be generated in the first place. There are some existing 3-D facial databases, such as BU-3DFE [14], Bosphorus [15], ZJU-3DFED [16], BU-4DFE [17], etc. The main reason behind creating these databases is for the research of identity recognition and facial expression analysis. However, to the best of our knowledge, there is no 3-D dynamic facial database which is currently available for the specific research on facial dysfunction for the clinical diagnosis. In order to fill this gap, Matuszewski et al. created a comprehensive 3-D dynamic facial database, Hi4D-ADSIP [18], using the Dimensional Imaging's latest 4-D capture system [19].

The database currently contains 3,360 facial sequences captured from 80 healthy volunteers (control subjects) of various age, gender and ethnicity. For each control subject, 42 facial articulations are recorded and 21 of them are captured for seven basic facial expressions at three different levels. These can be utilised for the relevant research of facial behaviour analysis, expression recognition, emotion detection, etc. The remaining articulations are specifically acquired to aid research into clinical diagnosis of facial dysfunctions.. In parallel to this control subject database, a clinical data set acquired from 43 stroke and Parkinson's disease patients has been established.

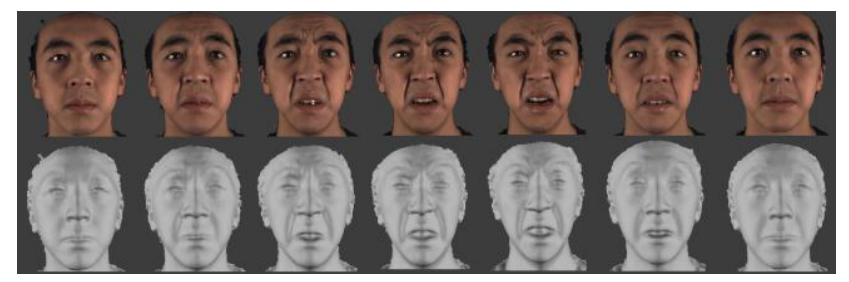

(a)

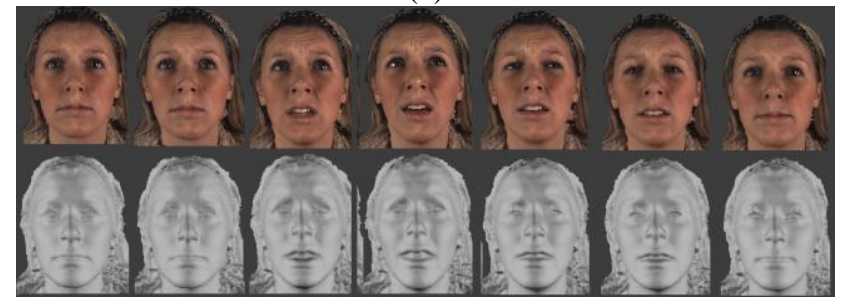

(b)

Figure 1. Samples of 3-D facial sequences in Hi4D-ADSIP database [18]: (a) male control subject, (b) female control subject

For each clinical subject, 18 specific facial articulations are recorded which correspond to some parts captured from the control subjects. Two examples of 3-D facial sequences in the Hi4D-ADSIP database (control subjects) are shown in Figure 1. More detail can be found in [18].

\section{FACE SEGMENTATION}

Prior to the symmetry plane extraction and asymmetry analysis of a face, the entire face region from the captured 3-D facial data needs to be extracted as it often contains occlusion such as, hair, accessories, etc. In order to locate the face region, it is critical to select a reference point. Since the nose tip is relatively reliable in terms of expression variations, it is chosen to be the reference point. The nose tip is determined by searching through the facial data to find the 3-D point with the greatest $z$-axis value. It is worth noting that a chin point could have a greater $z$-axis value than that of the nose tip in some rare occasions, however, since the nose is always above the chin, this problem is solved by using the geometric relationship between the nose and the chin, i.e. the nose is approximately positioned in the centre of a face while the chin is in the lower end of a face. This allows the proposed method to refine the result by excluding the chin point. For example, the proposed method can compare the two candidate points with the top two greatest $\mathrm{z}$-axis values, and take the one with a higher $\mathrm{y}$-axis value as the nose tip. As all facial data in the Hi4D-ADSIP database is frontally posed or very close, the relevant pose estimation can be neglected in this work. A very effective approach for achieving the frontal pose transformation can be found in [20].

Once the nose tip is determined, a sphere of radius $r$ centred at the nose tip is then used to segment the 3D face as shown in Figure 2 (a). A constant value of $r=130 \mathrm{~mm}$ is selected in this experiment to ensure a good area coverage of the face region even for the facial data with extreme expressions in the Hi4DADSIP database. This process crops an elliptical region (when 


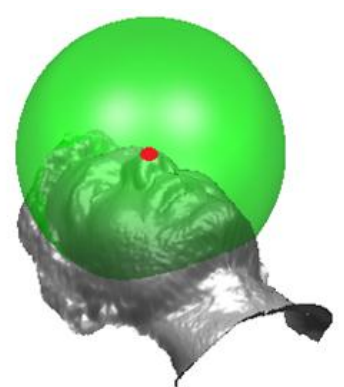

(a)

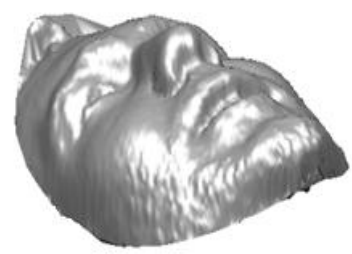

(b)
Figure 2. Example of the face segmentation: (a) nose tip with the predefined sphere, (b) segmented face region.

viewed in the $x y$-plane) from the face with vertical major axis and horizontal minor axis. Figure 2 (b) shows an example of a segmented 3-D face.

\section{SYMMETRY PLANE EXTRACTION}

The symmetry plane extraction is the most important part of the facial asymmetry analysis. From a correct symmetry plane of a human face, its asymmetry can be computed directly using its original data and the mirrored data obtained by a reflection across the symmetry plane extracted. Generally speaking, the entire symmetry plane extraction consists of three major processing stages, namely, mirror transformation, shape registration and symmetry profile extraction. While detection of the mirror transformation provides an initial alignment between the original data and its mirrored version, the shape registration is used to yield a refined match. Finally the symmetry profile extraction determines the symmetry plane of the face.

\section{A. Mirror Transformation}

The purpose of the mirror transformation is to initially estimate the mirrored version of the original facial data in order to extract the appropriate position of the symmetry plane later. It could also make the entire process of symmetry plane extraction automatic and pose-invariant. Following the work proposed by Benz et al. [12] and $\mathrm{Wu}$ et al. [21], the mirror transformation can be achieved by means of the principal component analysis (PCA).

Let $\mathbf{Q}$ denote a 3-D face which consists of a set of $N$ data points $\mathbf{q}_{i}\left(x_{i}, y_{i}, z_{i}\right)^{T} \in \mathrm{R}^{3}$, where $1 \leq i \leq N$, and $\mathbf{m}$ the centroid of the face expressed as a column vector. The covariance matrix of the data point distribution can be defined as follows:

$$
\mathbf{C}=\sum_{i=1}^{N}\left(\mathbf{q}_{i}-\mathbf{m}\right)\left(\mathbf{q}_{i}-\mathbf{m}\right)^{T}
$$

By applying the PCA, three eigenvalues, $\lambda_{1}, \lambda_{2}$ and $\lambda_{3}$, and their corresponding eigenvectors, $\mathbf{v}_{1}, \mathbf{v}_{2}$ and $\mathbf{v}_{3}$ of the covariance matrix $C$ can be obtained, where $\lambda_{1} \geq \lambda_{2} \geq \lambda_{3}$. Since the vertical dimension of human faces is typically longer than the horizontal dimension, the eigenvector $\mathbf{v}_{1}$ represents the vertical dimension of the faces, $\mathbf{v}_{2}$ associates with the horizontal dimension and $\mathbf{v}_{3}$ corresponds to the normal direction of the least-square fitted plane of the face [12]. Figure

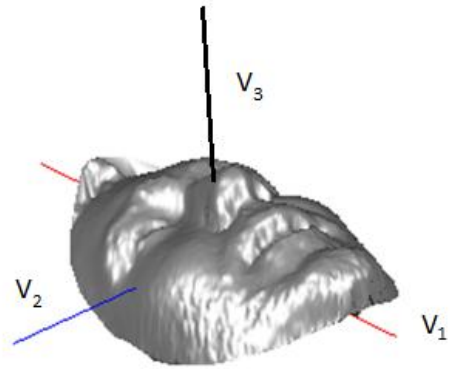

Figure 3. A 3-D face with three eigenvectors.

3 shows an example of the face and its relevant three eigenvectors in three-dimensional space.
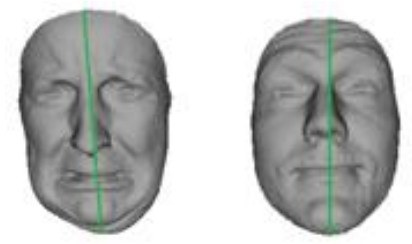

Figure 4. Results of the estimated mirror planes on 3-D faces

By taking $\mathbf{m}$ as the origin, a face can be transformed to a three-dimensional pose coordinate system (PCS) [22], with the $y$-axis matched to the eigenvector $\mathbf{v}_{1}$, the $z$-axis to $\mathbf{v}_{2}$, and the $x$ axis to $\mathbf{v}_{3}$. The coordinate system is then able to represent the head pose which mainly depends on the data distribution of the face. The $y z$-coordinate plane is considered as the mirror plane to obtain a mirrored face. This procedure can be described as:

$$
\begin{aligned}
& \mathbf{q}_{n i}=\mathbf{A} \cdot\left(\mathbf{q}_{i}-\mathbf{m}\right) \\
& \mathbf{q}_{m i}=\mathbf{R} \cdot \mathbf{A} \cdot\left(\mathbf{q}_{i}-\mathbf{m}\right)
\end{aligned}
$$

where $\mathbf{q}_{n i}$ is the original data point of the face which has been transformed to the PCS; $\mathbf{q}_{m i}$ is its mirrored data point in the PCS; $\mathbf{A}$ is the rotation matrix to the PCS and $\mathbf{R}$ is the reflection matric with respect to the mirror plane, and they are defined as:

$$
\begin{aligned}
& \mathbf{A}=\left[\begin{array}{lll}
v_{2}^{x} & v_{1}^{x} & v_{3}^{x} \\
v_{2}^{y} & v_{1}^{y} & v_{3}^{y} \\
v_{2}^{z} & v_{1}^{z} & v_{3}^{z}
\end{array}\right] \\
& \mathbf{R}=\left[\begin{array}{ccc}
-1 & 0 & 0 \\
0 & 1 & 0 \\
0 & 0 & 1
\end{array}\right]
\end{aligned}
$$

The results of the intersection between the estimated mirror plane and original faces from two individuals are shown in Figure 4. It can be seen that the mirror plane extracted using the PCA is close to the centre of the faces, i.e. the appropriate position of the actual symmetry plane, but not always accurate. This is mainly because the symmetry plane passes through the centroid of the object and is orthogonal to principal axes only if the object is bilaterally symmetric [23]. The human face itself is obviously not perfectly symmetric and also the boundary of the face is usually irregular which causes a strong asymmetry. 


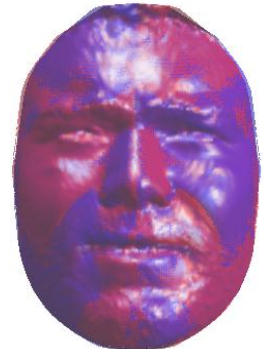

Figure 5. Result of the overlapped faces achieved by the ICP shape registration

\section{B. Shape Registration}

In order to extract the actual symmetry plane for a face, the misalignment between the original face and its mirror face provided by the mirror plane has to be minimised. This is often considered as the shape registration problem which can be achieved based on the iterative closest-point (ICP) algorithm [24], one of the most popular methods for the shape registration.

In general, the ICP algorithm consists of three iterative processing steps. The first step is to search dense point correspondences between the original and mirrored faces. The correspondence is determined based on the closest distance which is measured between points in the original and mirror faces using the Euclidean distance. The second step is to transform the mirrored face to match the original one using the rigid transformation calculated based on the estimated correspondence and minimise the cost function:

$$
\mathrm{E}=\frac{1}{N} \sum_{i=1}^{N}\left\|\mathbf{q}_{i}-\left(\mathbf{R} \mathbf{q}_{m i}^{\prime}+\mathbf{t}\right)\right\|
$$

where $N$ is the number of point correspondences in both faces; $\mathbf{q}_{i}$ is the data point in the original face and $\mathbf{q}_{m i}^{\prime}$ is the estimated correspondence in the mirrored face. $\mathbf{R}$ is a $3 \times 3$ rotation matrix and $\mathbf{t}$ is a $3 \times 1$ translation vector. The third step is to determine if the termination criterion is satisfied or go back to step one for the next iteration. A number of termination criteria can be used to stop the iteration: (i) the average distance between the point correspondences on two faces is below a fixed threshold, (ii) the change of the average distance between the point correspondences on two faces is below a fixed threshold, or (iii) a maximum number of iteration is reached. Figure 5 shows

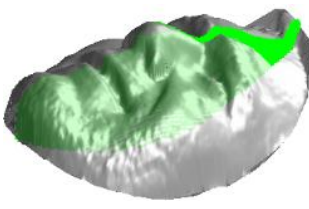

(a)

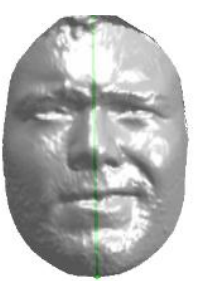

(b)

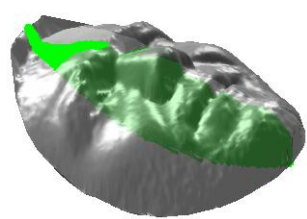

(c)
Figure 6. Example of extracted symmetry plane: (a) left view, (b) front view, (c) right view.

an example of the shape registration result achieved by the ICP. The red colour indicates the original face while the blue colour represents the mirrored one. It can be seen that the misalignment between two faces is minimised after the shape registration.

\section{Symmetry Profile Extraction}

After the original face and the mirrored face are matched, the symmetry plane can be directly extracted by fitting the midpoints, defined as

$$
\mathbf{q}_{c i}=\frac{1}{2}\left(\mathbf{q}_{i}+\mathbf{q}_{m i}^{\prime}\right), i=1,2, \ldots, N
$$

where $N$ is the number of points in the original face; $\mathbf{q}_{c i}$ are the midpoints which are used to fit a least-square plane. This fitted plane is the actual symmetry plane. The symmetry profile of a 3-D face is then extracted by intersecting the symmetry plane with the face itself. An example of the extracted symmetry plane from three viewpoints is shown in Figure 6.

Having the symmetry profile extracted, a number of facial feature points can be determined based on its geometrical information, and used for the asymmetry measurement later on. A small group of feature points are extracted in this work, such as inner eye corners, nose corners and nose tip. Since these feature points are located in the rigid regions of the human face and not affected by facial expressions, they can be precisely detected and used for other relevant applications, such as face segmentation, matching and registration [25].

A number of curvature-based methods can be applied for extracting the feature points on the face, and they are the principal curvature, the Gaussian curvature, the mean curvature and the HK segmentation. According to the experiments

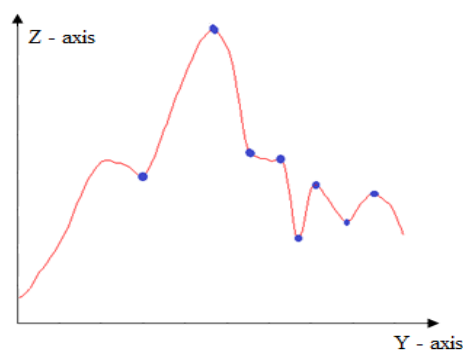

(a)

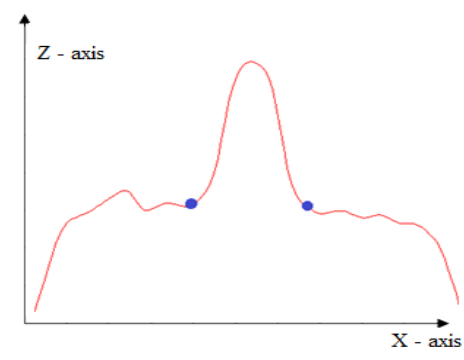

(b)

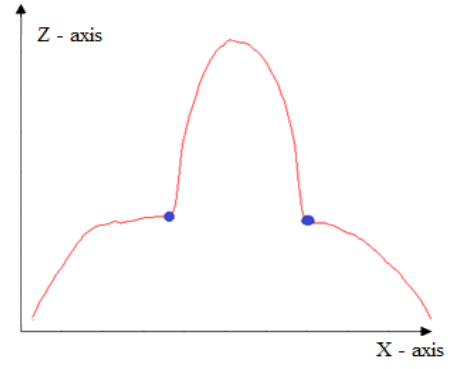

(c)

Figure 7. Examples of feature point extraction: (a) eight feature points, (b) inner eye corners, (c) nose corners. 
proposed by Segundo and Silva [25], the HK method is the most effective one to identify the feature points, which classifies points along a curve into nine different categories using the Gaussian curvature $(\mathrm{K})$ and mean curvature $(\mathrm{H})$. Based on the estimated categories, points of the curve can be then determined if they are the relevant feature points of interest. The method is also used for the feature point detection in this work. Using the contour of the symmetry profile, the HK method is able to identify eight feature points along the symmetry profile first which include nose tip, nose base, chin tip, etc., as shown in Figure 7 (a). Then the two inner eye corners can be identified using the horizontal slice across the face which intersects with the symmetry profile at the nose extracted from the symmetry profile and the same procedure is applied for detecting the two nose corners. Figure 7 (b) and (c) show the extracted inner eye corners and nose corners.

\section{ASYMMETRY MEASUREMENT}

Once the actual symmetry plane is determined, the asymmetry of the face can be measured by computing the average closest distance of all dense corresponding points between the original and mirrored faces. This is achieved by the ICP method [18]. In here, the mirrored face is obtained by reflection of the original face along the actual symmetry plane instead of the mirror plane. The Euclidean distance $d\left(\mathbf{q}, \mathbf{q}_{m}\right)$ between two points $\mathbf{q}(x, y, z)^{T}$ from the original face and $\mathbf{q}_{\mathrm{m}}\left(x_{m}\right.$, $\left.y_{m}, z_{m}\right)^{T}$ from the mirror face is defined as:

$$
d\left(\mathbf{q}, \mathbf{q}_{m}\right)=\sqrt{\left(x-x_{m}\right)^{2}+\left(y-y_{m}\right)^{2}+\left(z-z_{m}\right)^{2}}
$$

The bigger the value of the average closest distance, the higher level the facial asymmetry is. Figure 8 shows examples of the asymmetry measurement for four control subjects. It can be noticed how localised is the facial asymmetry and how does it evolve with the facial articulation. With the help of the extracted feature points, the face can be divided into a number of local regions. Therefore the facial asymmetry can be measured locally rather than on the whole face in order to fulfil various applications.

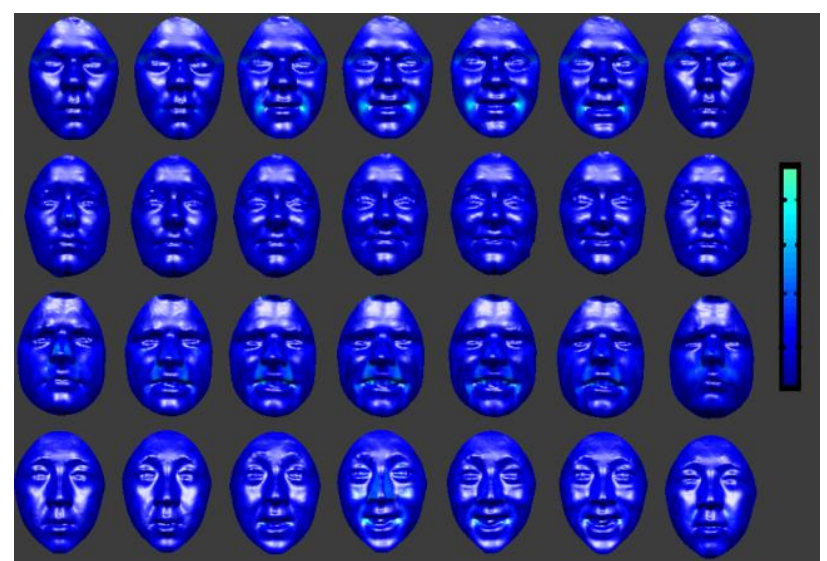

Figure 8. Examples of facial asymmetry measured with the colour of the surface representing normalised distance of the original face to the corresponding mirrored face.

\section{EVALUATION}

In order to evaluate the suitability of the proposed method for facial dysfunction measurement, a set of experiments were undertaken using the Hi4D-ADSIP database [18]. Since the database consists of 3-D facial sequences, the experiments mainly looked into the feasibility of dysfunction measurement in the dynamic sense rather than static, i.e. evolution of facial asymmetry over particular facial articulations. The facial sequences of 8 stroke patients were selected with four of them having two scanning sessions at approximately one month interval. Additionally 15 control subjects were also randomly chosen. Each 3-D face was divided into two sub-regions, namely, upper face and lower face, using the extracted feature points. The features used for assessment of facial dysfunction were extracted from the temporal evolution of the asymmetry measured during facial articulation. Examples of such temporal functions calculated from sequences for subjects pronouncing "You know how" phrase are shown in Figure 9 Based on the results reported by the authors in [18], the stroke patients were found to have not only higher facial asymmetry on average but also more significant changes in asymmetry measure during face articulation, a corresponding feature space is shown in Figure 10. As a result, the proposed method for asymmetry measurement is able to provide reliable information enabling not only recognition of the level of facial dysfunction, but also detection of minor changes in the asymmetry which might have not been noticed by clinicians during routine examination.

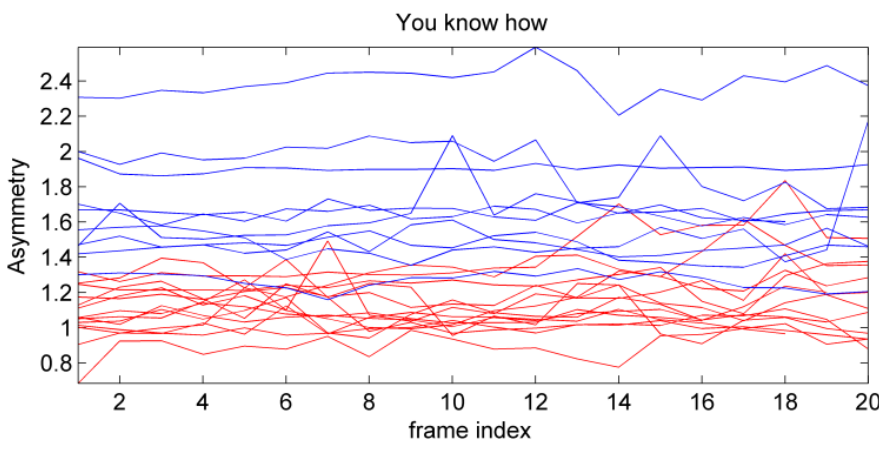

Figure 9. Example of asymmetry measured as function of facial articulation for the "You know how" sequence. Data for control subjects and stroke patients are represented by red and blue lines respectively.

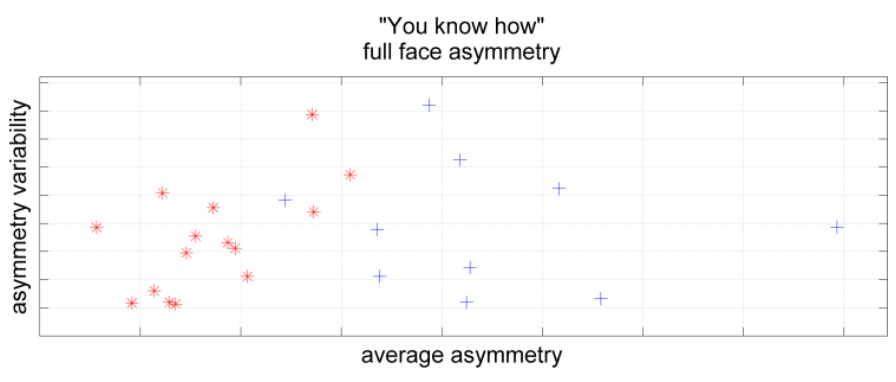

Figure 10. Two dimensional feature space representing asymmetry for "You know how" sequences. Each point represents single sequence with red '*' and blue '+' depicting control and clinical subjects respectively. 


\section{CONCLUSIONS AND FUTURE WORK}

This paper describes a novel method for measuring facial asymmetry using dynamic 3-D scans of human faces. The proposed method is invariant to arbitrary pose and variation of facial expressions. It is also able to extract a set of feature points on the 3-D face which can be used for segmenting the face into local regions. The method was tested on a set of preliminary experiments using the Hi4D-ADSIP database with the comparison between stroke patients and healthy individuals. The experimental results indicate that the proposed method has the potential for detecting the facial dysfunction. This encouraged the authors to extend its applications to other conditions, such as Parkinson's disease. In order to achieve these objectives, a more comprehensive test based on the Hi4D-ADSIP database and validation with clinical assessment data are currently undertaken at the ADSIP Research Centre.

\section{ACKNOWLEDGMENTS}

This work was supported by the Engineering and Physical Sciences Research Council (EPSRC) [grant number EP/H024913/1].

\section{REFERENCES}

[1] L. Yin, X. Wei, P. Longo, and A. Bhuvanesh, "Facial Expression Using Intensity-Variant 3D Data for Human Computer Interaction", International Conference on Pattern Recognition, pp.1248-1251, 2006.

[2] C. L. Lisetti, and D. J. Schiano, "Automatic Facial Expression Interpretation: Where Human-Computer Interaction, Artificial Intelligence and Cognitive Science Intersect", Pragmatics and Cognition (Special Issue on Facial Information Processing: Multidisciplinary Perspective), vol.8 (1), pp.185-235, 2000.

[3] G. J. Edwards, T. F. Cootes, C. J. Taylor, "Face Recognition Using Active Appearance Models", Proceeding of the 5th European Conference on Computer Vision, vol.2, pp.581-595, 1998.

[4] J. Wang, L Yin, X. Wei, and Y. Sun, “3D Facial Expression Recognition Based on Primitive Surface Feature Distribution", IEEE CVPR, pp.1726, 2006.

[5] S. Brahnam, C. Chuang, F. Y. Shih, and M. R. Slack, "Machine Recognition and Representation of Neonatal Facial Displays of Acute Pain”, Artificial Intell. in Medicine, vol.36 (3), pp. 211-222, 2006

[6] P. Meyer-Marcotty, G. W. Alpers, A. B. M. Gerdes and A. StellzigEisenhauer, "Impact of facial asymmetry in visual perception: a 3dimenisonal data analysis", American Journal of Orthodontics and Dentofacial Orthopedics, vol.137(2), pp.1-8, 2010.

[7] G. Marola, "On the detection of the axes of symmetry of symmetric and almost symmetric plannar images", IEEE Transaction on Pattern Recognition and Machine Intelliegence, vol.11, pp. 104-108, 1998

[8] T. Masuda, K. Yamamoto and H. Yamada, "Detection of partial symmetry using correlation with rotated-reflected images", Pattern Recognition, vol.26(8), pp.1245-1253, 1993.
[9] N. Kiryati and Y. Gofman, "Detecting symmetry in grey level images: the global optimisation approach", International Journal of Computer Vision, vol.29, pp.29-45, 1998.

[10] C. Sun and J. Sherrah, "3-D symmetry detection using the extended gaussian image", IEEE Transaction on Pattern Analysis and Machine Intelligence, vol.19(2), pp.164-168, 1997.

[11] G. Pan, Y. Wang, Y. Qi and Z. Wu, "Find symmetry plane of 3D face shape", IEEE International Conference on Pattern Recognition, pp. 1143-1146, 2006

[12] M. Benz, X. Laboureux, T. Maier, E. Nkenke, S. Seeger, F. W. Neukam and G. Hausler, "The symmetry of faces", Proceeding of the Vision, Modeling, and Visualisation Conference 2002 (VMV 2002), pp. 43-50, 2002

[13] W. Quan, B. J. Matuszewski, L.-K. Shark and D. Ait-Boudaoud, "Facial expression biometrics using statistical shape models", EURASIP Journal on Advances in Signal Processing, SpringerOpen, vol.2009, pp. 1-17, 2009.

[14] L. Yin, X. Wei, Y. Sun, J. Wang, and M. Rosato, "A 3D facial expression database for facial behaviour research", $7^{\text {th }}$ International Conference on Automatic Face and Gesture Recognition, pp. 211-216, 2006.

[15] A. Savran, N. Alyuz, H. Dibeklioglu, O. Celiktutan, B. Gokberk. B. Sankur and L. Akarun, "Bosphorus database for 3D face analysis", First COST Workshop on Biometrics and Identity Management (BIOID 2008), 2008.

[16] Y. Wang, G. Pan, Z. Wu and Y. Wang, "Exploring facial expression effects in 3D face recognition using partial ICP", Lecture Note in Computer Sciences, vol.3851(2006), pp. 581-590, 2006

[17] L. Yin, X. Chen, Y. Sun, T. Worm and M. Reale, "A high-resolution 3D dynamic facial expression database", $8^{\text {th }}$ International Conference on Automatic Face and Gesture Recognition (FGR08), 2008.

[18] B. J. Matuszewski, W. Quan, L.-K. Shark, A. S. McLoughlin, C. E. Lightbody, H. C. A. Emsley, C. L. Watkins, "Hi4D-ADSIP 3D dynamic facial articulation database", Image and Vision Computing, DOI: 10.1016/J.IMAVIS.2012.02.002, 2012.

[19] Dimensional Imaging, http://www.di3d.com , 2011.

[20] Y. Sun and L. Yin, " Automatic pose estimation of 3D facial models", IEEE Interantional Conference on Pattern Recognition (ICPR), 2008.

[21] Y. Wu, G. Pan and Z. Wu, "Face authentication based on multiple profiles extracted from range data", Audio and Video-based Biometric Person Authentication, Lecture Notes in Computer Sciences, vol. 2688(2003), pp.515-522, Springr-Verlag, 2003.

[22] L. Zhang, A. Razdan, G. Farin, J. Femiani, M. Bae and C. Lockwood, "3D face authentication and recognition based on bilateral symmetry analysis". Visual Computer, vol. 22(1), pp. 43-55, 2006.

[23] X. M. Tang, J. S. Chen and Y. S. Moon, "Accurate 3D face registration on the symmetry plane analysis on nose regions", $16^{\text {th }}$ European Signal Processing Conference (EUSIPCO 2008), 2008

[24] P. J. Besl and N. D. McKay, "A method for registration of 3-D shapes", IEEE Transcations on Pattern Analaysis and Machine Intelligence, vol.14(2), pp. 239-256, 1992.

[25] M. P. Segundo and L. Silva, "Automatic face segmentation and facial landmark detection in range images", IEEE Transaction on Systems, Man and Cybernetics, vol.40(5), pp. 1319-1330, 2010. 